Morphological brain changes and CC size were evaluated using standard MRI sequences. The MRI evaluators were not informed about the results of visual examinations.

Results Impaired visual acuity was detected in 9/12 cases with abnormal CC (75\%) and in 10\% of children with normal CC (p $<0.01)$. There was a significant correlation between the CC size and Frostig test results (abnormal CC group vs. normal CC group: 91 vs. 80.7 points; $p=0.03$ adjusted for history of ROP). Absence of stereoscopic vision was more frequent in the group of abnormal CC (7/12 vs. $2 / 20 ; p=0.03)$. The frequency of abnormal VEP was similar in the both groups.

Conclusion A strong correlation between vision impairment and CC size was observed. This suggests that CC plays important function in integration of visual perception.

Study supported by National Science Centre, Poland: grant number: 2011/03/B/NZ5/05678.

\section{P0-0416 2ND TRIMESTER HEAD SIZE IN FETUSES WITH CONGENITAL HEART DISEASE: A COHORT STUDY}

${ }^{1} \mathrm{MH}$ Lauridsen, ${ }^{2} \mathrm{OB}$ Petersen, ${ }^{3} \mathrm{EM}$ Vestergaard, ${ }^{1} \mathrm{~TB}$ Henriksen, ${ }^{1} \mathrm{JR}$ Østergaard, ${ }^{1} \mathrm{NB}$ Matthiesen, ${ }^{4}$ VE Hjortdal. ${ }^{1}$ Paediatrics, Aarhus University Hospital, Aarhus N, Denmark; ${ }^{2}$ Obstetrics and Gynaecology, Aarhus University Hospital, Aarhus N, Denmark; ${ }^{3}$ Clinical Genetics, Aarhus University Hospital, Aarhus N, Denmark; ${ }^{4}$ Cardio-Thoracic Surgery, Aarhus University Hospital, Aarhus N, Denmark

\subsection{6/archdischild-2014-307384.1061}

Background and aims Congenital heart disease (CHD) is associated with neuro-developmental disorder. The influence of CHD on the brain is believed to begin during pregnancy. The aim of this study is to describe a 2-year cohort of fetuses with major and minor $\mathrm{CHD}$ and to investigate if and when during pregnancy cerebral growth is disrupted. We hypothesise that fetal cerebral growth is impaired as early as 2 nd trimester.

Method Pregnant women in Denmark (more than 95\%) attend two publicly funded ultrasound scans; at 12 weeks gestational age (GA) and at 19-20 weeks GA. Fetal biometrics and abnormal ultrasound findings are registered. Fetuses in Western Denmark (2.9 mill inhabitants) screened between January 1st 2012 and December 31st 2013, diagnosed with any structural, nonsyndromic CHD either during pregnancy or up to six months after birth, are included in the study.

Results 129 fetuses with CHD were identified prenatally. Ninety-eight (76\%) were genetically screened, primarily by chromosomal micro-array analysis $(n=72)$. Nineteen pregnant women $(15 \%)$ declined invasive testing. Twenty-three fetuses $(18 \%)$ were excluded due to genetic syndromes, mainly aneuploidies $(\mathrm{n}=14)$ and seven $(5 \%)$ were excluded due to extra-cardiac malformations. Ninety-nine fetuses $(77 \%)$ with presumed non-syndromic CHD were included. Head circumference in week 19-20 was significantly smaller than average with a mean $\mathrm{z}$ score of -0.4 (95\% CI: $-0.7,-0.2)(\mathrm{p}<0.01)$. Analyses are undergoing and results will be presented at the meeting.

Conclusions Preliminary unadjusted results suggest that fetal cerebral growth in children with CHD may be disrupted as early as 2nd trimester.

\section{P0-0417 WITHDRAWN}

\section{PO-0418 BRAIN PROCESSING OF MUSIC IN THE NEWBORNS}

${ }^{1} \mathrm{~L}$ Lordier, ${ }^{2} \mathrm{~F}$ Grouiller, ${ }^{1} \mathrm{~A}$ Van der veek, ${ }^{3} \mathrm{D}$ Grandjean, ${ }^{2} \mathrm{~F}$ Lazeyras, ${ }^{1} \mathrm{PS}$ Hüppi. ${ }^{1}$ Division of Development and Growth Department of Pediatrics, HUG, Geneva, Switzerland; ${ }^{2}$ Department of Radiology and Medical Informatics, University of Geneva, Geneva, Switzerland; ${ }^{3}$ Neuroscience of Emotion and Affective Dynamics Lab Department of Psychology, University of Geneva, Geneva, Switzerland

\subsection{6/archdischild-2014-307384.1062}

In newborns, some basic music processing seems to be present few days after birth (1). Neonatal intensive care units are willing to use music to enrich preterm infant environment. Indeed, researches in neonatal intensive care units showed music impacts on physiological and behavioural responses, caloric intake, and energy expenditure of premature babies (2). The aim of this study is to understand how music is processed in newborns and if premature birth impacts on this processing.

Twenty-four healthy newborns (14 preterm scanned at term equivalent age and 10 full-term infants) have been recruited. Infants underwent functional MRI (fMRI) at 3T during natural sleep or while resting quietly in the scanner without any sedation. FMRI data were realigned; coregistered; normalised to a T2 neonatal template; and smoothed. Random-effect analyses have been done to observe the group activation on all newborns and to compare term and preterm newborns.

At the group level, we observed bilateral activation of auditory regions. At the cluster level, the right auditory cortex ( $\mathrm{p}=$ 0.028 , corrected for multiple comparison, extent $=70$ voxels) was more activated than the left auditory cortex $(\mathrm{p}=0.067$, corrected for multiple comparison, extent $=52$ voxels) during music. Furthermore, no difference was found between full-term and premature groups.

Our preliminary results show functional asymmetry in auditory cortex already present at 40weeks gestational age. Those results corroborate with those found in adults (3), in processing pitch changes in 3-to-6 month's old children (4) and in full-term newborns (1). More analyses are needed to explore further music processing differences in these two populations.

\section{PO-0419 INCIDENCE OF CEREBRAL OXYGEN DESATURATION AMONG NEONATES UNDERGOING GENERAL ANAESTHESIA}

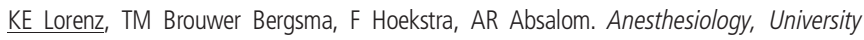
Medical Center Groningen (UMCG), Groningen, Netherlands

\subsection{6/archdischild-2014-307384.1063}

Background Cerebral autoregulation (CA) is a physiologic mechanism ensuring constant blood flow to the brain independent of changes (within physiological limits) in mean systemic blood pressures. Compromised CA can lead to ischemia, associated with hypoxic injury and long term sequelae. The efficiency of $\mathrm{CA}$ in neonates is not well-known, especially during general anaesthesia. We aimed to determine incidences of significant cerebral desaturation $\left(\mathrm{S}_{\mathrm{c}} \mathrm{O}_{2}\right)$.

Methods Observational data were collected from 33 premature and term neonates (up to post-menstrual age 44 weeks), receiving general anaesthesia for surgery. Near- infrared spectroscopy (ForeSight ${ }^{\circledR}$ CerebralOximeter, CASMED, USA) was used to noninvasively measure regional $\mathrm{S}_{\mathrm{c}} \mathrm{O}_{2}$. When used, invasive arterial pressures were recorded electronically. We then calculated the 